\title{
Characterization of zwitterion-modified poly(amidoamine) dendrimers in aqueous solution via a thorough NMR investigation
}

\author{
Jinyuan Liu ${ }^{1,2}$, Zhijuan Xiong ${ }^{2}$, Mingwu Shen ${ }^{2}$, Istvan Banyai ${ }^{3, a}$, and Xiangyang Shi ${ }^{1,2,4, b}$ \\ 1 Department of Interventional and Vascular Surgery, Shanghai Tenth People's Hospital, Tongji University School of Medicine, \\ Shanghai 200072, China \\ 2 College of Chemistry, Chemical Engineering and Biotechnology, Donghua University, Shanghai 201620, China \\ 3 Department of Physical Chemistry, University of Debrecen, H-4032 Debrecen, Hungary \\ 4 CQM - Centro de Química da Madeira, Universidade da Madeira, Campus da Penteada, 9020-105 Funchal, Portugal
}

Received 9 November 2019 and Received in final form 17 December 2019

Published online: 4 February 2020

(C) The Author(s) 2020. This article is published with open access at Springerlink.com

\begin{abstract}
Zwitterions are a class of unique molecules that can be modified onto nanomaterials to render them with antifouling properties. Here we report a thorough NMR investigation of dendrimers modified with zwitterions in terms of their structure, hydrodynamic size, and diffusion time in aqueous solution. In this present work, poly(amidoamine) (PAMAM) dendrimers of generation 5 (G5) were partially decorated with carboxybetaine acrylamide (CBAA), 2-methacryloyloxyethyl phosphorylcholine (MPC), and 1,3-propane sultone (1,3-PS), respectively with different modification degrees. The formed zwitterionmodified G5 dendrimers were characterized using NMR techniques. We show that the zwitterion modification leads to increased G5 dendrimer size in aqueous solution, suggesting that the modified zwitterions can form a hydration layer on the surface of G5 dendrimers. In addition, the hydrodynamic sizes of G5 dendrimers modified with different zwitterions but with the same degree of surface modification are discrepant depending on the type of zwitterions. The present study provides a new physical insight into the structure of zwitterion-modified G5 dendrimers by NMR techniques, which is beneficial for further design of different biomedical applications.
\end{abstract}

\section{Introduction}

Reducing the adhesion of environmental molecules to surfaces has long been a goal of applied surface science. The most active areas at the biological interface are to render the particles with improved antifouling property [1]. Zwitterion is a kind of material that possesses moieties with both cationic and anionic groups, allowing for the formation of a thick hydration layer onto the particle surface after the particles are coated with them $[2-5]$. The zwitterion-coated particles can be rendered with excellent antifouling property to resist the non-specific protein adsorption, to escape the recognition of the reticuloendothelial system (RES) after systemic injection, and to prolong the blood circulation time in the body for enhanced accu-

\footnotetext{
* Contribution to the Topical Issue "Branching Dynamics at the Mesoscopic Scale" edited by Yongsheng Han, Hui Xing, Dongke Sun.

${ }^{a}$ e-mail: banyai.istvan@science.unideb.hu (corresponding author)

b e-mail: xshi@dhu.edu.cn (corresponding author)
}

mulation in the disease site [6-11]. At present, the commonly studied zwitterion types include phosphoric acid choline (PC) $[12,13]$, carboxylic acid betaine (CB) [14, $15]$, and sulfonic acid betaine (SB) $[16,17]$, etc.

The main methods to characterize the antifouling properties of zwitterion-modified nanoparticles (NPs) include nonspecific protein adsorption test, macrophage cellular uptake assay in vitro and pharmacokinetics in vivo [18-20]. All of these detection methods require a complicated equipment, and cell and animal experiments, which require specialized experimental skills and instruments to obtain reliable experimental data. In addition to the above characterization techniques, the structural characterization of zwitterion-modified materials can be usually realized through NMR and/or Fourier transform infrared (FTIR) spectroscopy. However, in most cases, besides the above-mentioned bio-based techniques, currently the physical characterization of zwitterion-modified nanoparticles in aqueous solution is still lacking.

Recently, with the rapid development of NMR techniques, high-resolution NMR spectroscopy, NMR diffusiometry, and NMR cryoporometry have been used to 


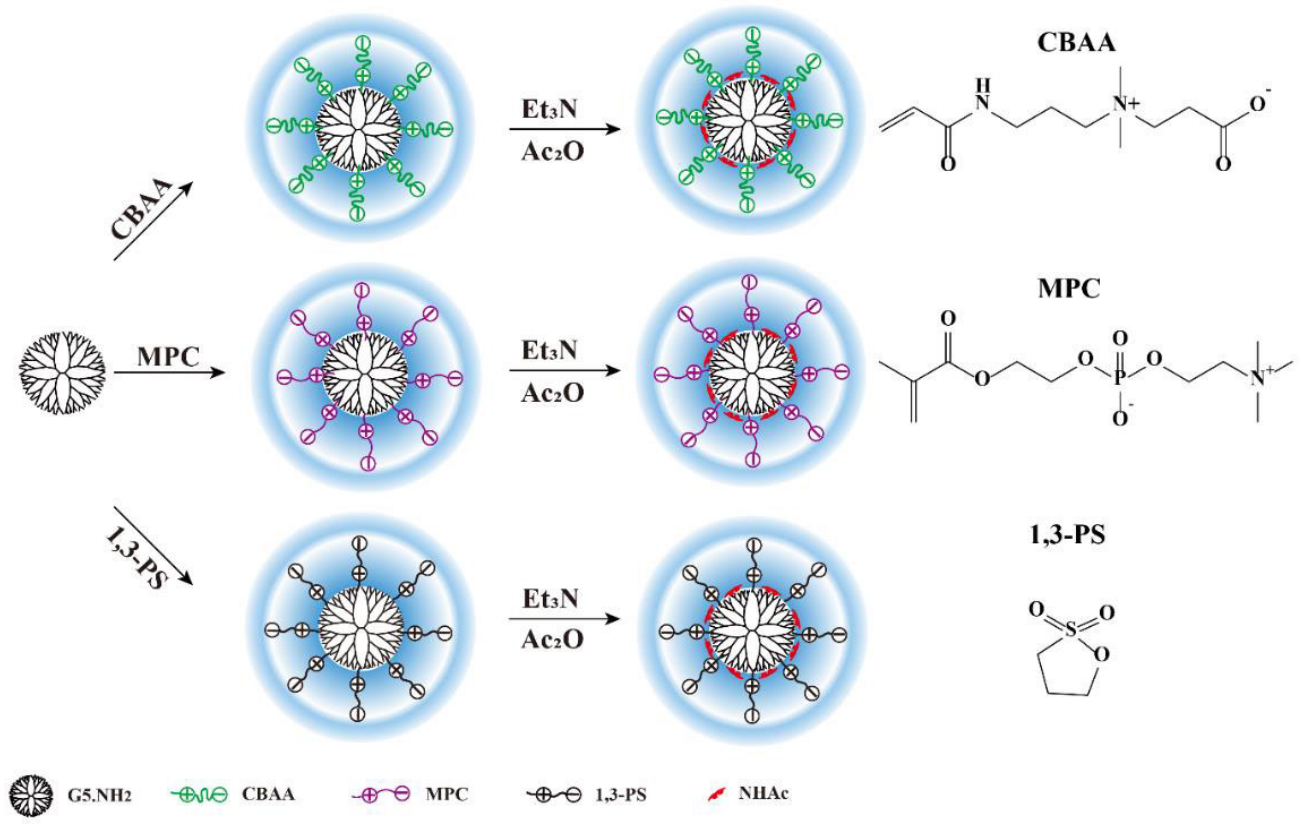

Fig. 1. Schematic representation of the preparation of CBAA-, MPC- or 1,3-PS-modified G5 dendrimer. Et ${ }_{3} \mathrm{~N}_{\text {and }} \mathrm{Ac}_{2} \mathrm{O}$ denote triethylamine and acetic anhydride, respectively.

characterize NPs in aqueous solution [21-26]. For instance, in our previous work [26], we used NMR techniques to characterize the internal structure of polyethylenimine (PEI)-entrapped gold nanoparticles and disclosed the strong interaction existing between the Au NPs and the innermost PEI, providing evidence to demonstrate that $\mathrm{Au}$ NPs are entrapped within an individual polymer instead of being stabilized. In another work [27], we employed NMR diffusiometry to examine the diffusion rate and hydrodynamic size of dendrimer/carbon dot nanhybrid materials to confirm the interaction between the multifunctional dendrimers and the carbon dots. These studies underscore the importance to use advanced NMR techniques to delineate functional dendrimers and hybrid materials.

In this present study, we prepared three representative zwitterionic dendrimers by reacting carboxybetaine acrylamide (CBAA), 2-methacryloyloxy ethyl phosphorylcholine (MPC) and 1,3-propanesultone (1,3-PS) with generation 5 (G5) poly(amidoamine) (PAMAM) dendrimers with different modification degrees (fig. 1). ${ }^{1} \mathrm{H}$ NMR, 2D COSY, 2D DOSY and diffusion NMR were used to characterize the structure of the synthesized dendrimers and to calculate the hydrodynamic sizes of the dendrimers in aqueous solution. The obtained results are beneficial for a better understanding of the physical structure of the zwitterionic dendrimers for different biomedical applications.

\section{Experimental}

\subsection{Materials}

Ethylenediamine core amine-terminated G5 PAMAM dendrimers $\left(\mathrm{G} 5 . \mathrm{NH}_{2}\right)$ were purchased from Dendritech (Midland, MI). 2,6-Bis(1,1-dimethylethyl)-4-methylphenol
(BHT), $\quad \beta$-propiolactone $\quad$ (98\%), 2-methacryloyloxy ethyl phosphorylcholine (MPC) and 1,3-propanesultone (1,3-PS) were from J\&K Scientific (Shanghai, China). Acetic anhydride, triethylamine, $\mathrm{HAuCl}_{4} \cdot 4 \mathrm{H}_{2} \mathrm{O}$ and all the other chemicals and solvents were obtained from Sinopharm Chemical Reagent Co., Ltd. (Shanghai, China). Water used in all experiments was purified using a Milli-Q Plus 185 water purification system (Millipore, Bedford, MA) to have a resistivity higher than 18.2 $\mathrm{M} \Omega \cdot \mathrm{cm}$. Regenerated cellulose dialysis membranes with a molecular weight cut-off (MWCO) of 10000 were acquired from Fisher (Pittsburgh, PA).

\subsection{Synthesis of carboxybetanie acrylamide (CBAA)-, MPC-, and 1,3-PS-modified G5 dendrimers}

CBAA was first synthesized according to our previous work [28]. G5 PAMAM dendrimers were partially modified by CBAA via Michael addition reaction according to protocols reported in the literature with slight modification [28]. In brief, CBAA (20 mg) dissolved in an aqueous $\mathrm{NaCl}$ solution $(0.138 \mathrm{M}, 5 \mathrm{~mL})$ was dropwise added into a methanol solution of $\mathrm{G} 5 . \mathrm{NH}_{2}$ dendrimers $(10 \mathrm{mg}$, $5 \mathrm{~mL}$ ) under stirring at room temperature. After $72 \mathrm{~h}$, the mixture was dialyzed against phosphate buffered saline (PBS, 3 times, $4 \mathrm{~L}$ ) and water $(6$ times, $4 \mathrm{~L})$ using a dialysis membrane with an MWCO of 10000 for $48 \mathrm{~h}$, and then lyophilized to get the product of $\mathrm{G} 5 . \mathrm{NH}_{2}-\mathrm{CBAA}_{20}$.

G5. $\mathrm{NH}_{2}$ dendrimers were also partially modified with $\mathrm{MPC}$ via Michael addition and 1,3-PS via a one-step ringopening reaction according to our previous work [29]. Accordingly, the generated $\mathrm{G} 5 . \mathrm{NH}_{2}-\mathrm{CBAA}_{80}, \mathrm{G} 5 . \mathrm{NH}_{2}$ $\mathrm{MPC}_{20}, \mathrm{G} 5 . \mathrm{NH}_{2}-\mathrm{MPC}_{80}, \mathrm{G} 5 . \mathrm{NH}_{2}-\mathrm{PS}_{20}$, and $\mathrm{G} 5 . \mathrm{NH}_{2}-$ $\mathrm{PS}_{80}$ products are listed in table 1. 
Table 1. Synthesis of CBAA-, MPC-, and 1,3-PS-modified G5 dendrimers.

\begin{tabular}{|c|c|c|}
\hline Reactant & Reactant & Product \\
\hline G5. $\mathrm{NH}_{2}(10 \mathrm{mg}$, in $5 \mathrm{~mL}$ methanol $)$ & CBAA $(20 \mathrm{mg}$, in $\mathrm{NaCl}$ solution $(0.138 \mathrm{M}, 5 \mathrm{~mL}))$ & G5. $\mathrm{NH}_{2}-\mathrm{CBAA}_{20}$ \\
\hline G5. $\mathrm{NH}_{2}(10 \mathrm{mg}$, in $5 \mathrm{~mL}$ methanol $)$ & CBAA $(65 \mathrm{mg}$, in $\mathrm{NaCl}$ solution $(0.138 \mathrm{M}, 5 \mathrm{~mL}))$ & G5. $\mathrm{NH}_{2}-\mathrm{CBAA}_{80}$ \\
\hline G5. $\mathrm{NH}_{2}(10 \mathrm{mg}$, in $5 \mathrm{~mL}$ water $)$ & MPC $(26.5 \mathrm{mg}$, in $5 \mathrm{~mL}$ water $)$ & $\mathrm{G} 5 . \mathrm{NH}_{2}-\mathrm{MPC}_{20}$ \\
\hline G5. $\mathrm{NH}_{2}(10 \mathrm{mg}$, in $5 \mathrm{~mL}$ water $)$ & $\mathrm{MPC}(83.5 \mathrm{mg}$, in $5 \mathrm{~mL}$ water $)$ & G5. $\mathrm{NH}_{2}-\mathrm{MPC}_{80}$ \\
\hline G5. $\mathrm{NH}_{2}(10 \mathrm{mg}$, in $5 \mathrm{~mL}$ water $)$ & 1,3 -PS $(1.2 \mathrm{mg}$, in $5 \mathrm{~mL}$ water $)$ & $\mathrm{G} 5 . \mathrm{NH}_{2}-\mathrm{PS}_{20}$ \\
\hline G5. $\mathrm{NH}_{2}(10 \mathrm{mg}$, in $5 \mathrm{~mL}$ water $)$ & 1,3 -PS $(3.8 \mathrm{mg}$, in $5 \mathrm{~mL}$ water $)$ & G5. $\mathrm{NH}_{2}-\mathrm{PS}_{80}$ \\
\hline
\end{tabular}

Table 2. Synthesis of CBAA-, MPC-, and 1,3-PS-modified G5 dendrimers with remaining dendrimer terminal amines being acetylated.

\begin{tabular}{|c|c|c|c|}
\hline Reactant & Triethylamine & Acetic anhydride & Product \\
\hline 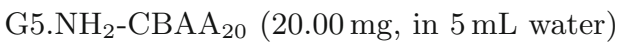 & $116.40 \mu \mathrm{L}$ & $79.16 \mu \mathrm{L}$ & G5.NHAc-CBAA $_{20}$ \\
\hline 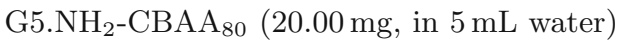 & $80.41 \mu \mathrm{L}$ & $54.69 \mu \mathrm{L}$ & G5.NHAc-CBAA 80 \\
\hline G5. $\mathrm{NH}_{2}-\mathrm{MPC}_{20}(20.00 \mathrm{mg}$, in $5 \mathrm{~mL}$ water $)$ & $115.51 \mu \mathrm{L}$ & $75.84 \mu \mathrm{L}$ & G5.NHAc-MPC ${ }_{20}$ \\
\hline G5. $\mathrm{NH}_{2}-\mathrm{MPC}_{80}(20.00 \mathrm{mg}$, in $5 \mathrm{~mL}$ water $)$ & $71.73 \mu \mathrm{L}$ & $48.78 \mu \mathrm{L}$ & G5.NHAc-MPC 80 \\
\hline G5. $\mathrm{NH}_{2}-\mathrm{PS}_{20}(20.00 \mathrm{mg}$, in $5 \mathrm{~mL}$ water $)$ & $125.07 \mu \mathrm{L}$ & $85.06 \mu \mathrm{L}$ & G5.NHAc-PS 20 \\
\hline G5. $\mathrm{NH}_{2}-\mathrm{PS}_{80}(20.00 \mathrm{mg}$, in $5 \mathrm{~mL}$ water $)$ & $99.48 \mu \mathrm{L}$ & $67.65 \mu \mathrm{L}$ & G5.NHAc-PS 80 \\
\hline
\end{tabular}

Next, to neutralize the remaining dendrimer terminal primary amines, the product of $\mathrm{G} 5 . \mathrm{NH}_{2}-\mathrm{CBAA}_{20}$ was added with triethylamine $(60.64 \mu \mathrm{L})$ under stirring for $0.5 \mathrm{~h}$, followed by addition of acetic anhydride $(34.34 \mu \mathrm{L})$ while stirring $12 \mathrm{~h}$. The reaction mixture was dialyzed and lyophilized according to protocols described in the literature [29] to generate G5.NHAc-CBAA 20 . Under similar reaction and purification conditions, products of G5.NHAc-CBAA 80 , G5.NHAc-MPC 20 , G5.NHAc$\mathrm{MPC}_{80}$, G5.NHAc-PS 20 , and G5.NHAc-PS 80 were also produced (table 2).

\subsection{NMR techniques}

All samples were dissolved in deuterium oxide $\left(\mathrm{D}_{2} \mathrm{O}\right) \cdot{ }^{1} \mathrm{H}$ NMR, diffusion ordered spectroscopy (DOSY), and correlation spectroscopy (COSY) and diffusion time were analyzed by a Bruker DRX 400 NMR spectrometer (Karlsruhe, Germany) according to the standard pulse programs [26]. The diffusion coefficient was calculate according to $[26]$

$$
I=I_{0} \exp \left(-D_{o b s}(\Delta-\delta / 3) \gamma^{2} G^{2} \delta^{2}\right),
$$

where $G$ is the gradient field strength (in gauss/cm), increasing with 32 square distant steps from 0 to approximately $50 \mathrm{G} \mathrm{cm}^{-1} ; I$ is the integral of the peak area at a given value $G ; I_{0}$ is the integral of the peak area at $G=0$; $\gamma$ is the magnetogyric constant of the nucleus $(2.675 \times$ $10^{8} \mathrm{~T}^{-1} \mathrm{~s}^{-1}$ for $\left.{ }^{1} \mathrm{H}\right) ; \delta$ is the diffusion gradient length parameter $(4.0 \mathrm{~ms}) ; \Delta$ is the diffusion delay $(100.0 \mathrm{~ms})$, and the non-linear least squares method was used to determine the diffusion coefficient; $D_{o b s}$ is in individual units and $D_{o b s} \gamma^{2}$ was calculated after fitting the exponential curve (1) at the measured echo intensity $(I)$ as a function of $G^{2}$ determined by the number of experiments. The real diffusion coefficient $D$ was calculated as $\kappa D_{o b s} \gamma^{2}$, where $\kappa$ is the calibration constant of the gradient. MestReNova 6.1 software was used for post processing.

\section{Results and discussion}

\section{$3.1{ }^{1} \mathrm{H}$ NMR spectroscopy}

${ }^{1} \mathrm{H}$ NMR spectroscopy was first performed to confirm the modification of CBAA, MPC, and 1,3-PS onto the surface of G5 PAMAM dendrimers and the modification degree (fig. 2). For G5. $\mathrm{NH}_{2}-\mathrm{CBAA}_{20}$ and $\mathrm{G} 5 \cdot \mathrm{NH}_{2}-\mathrm{CBAA}_{80}$, by comparison of the $\mathrm{CBAA}-\mathrm{CH}_{2}$ - proton peak at $1.86 \mathrm{ppm}$ with the G5 methylene protons [28], the number of CBAA coupled to each G5 dendrimer was calculated to be 19 and 78 , respectively. Similarly, by comparison of the methyl proton of MPC at $0.97 \mathrm{ppm}$ with dendrimer methylene protons [30], the $\mathrm{G} 5 . \mathrm{NH}_{2}-\mathrm{MPC}_{20}$ and $\mathrm{G} 5 . \mathrm{NH}_{2}-\mathrm{MPC}_{80}$ were calculated to have 20 and $79 \mathrm{MPC}$ moieties linked onto each dendrimer, respectively. Furthermore, the comparison of the characteristic methylene protons of 1,3-PS at $1.88 \mathrm{ppm}$ with those of G5 dendrimers led us to conclude that the number of 1,3-PS attached to each G5 dendrimer was 20 and 80 [31], respectively for the $\mathrm{G} 5 . \mathrm{NH}_{2}$ $\mathrm{PS}_{20}$ and $\mathrm{G} 5 . \mathrm{NH}_{2}-\mathrm{PS}_{80}$.

The above products were then acetylated to shield the residue dendrimer terminal amines according to the literature [32]. Obviously, the peaks at $1.8 \mathrm{ppm}$ associated to the acetyl groups appear, validating the success of the acetylation reaction (fig. 3). Although there is somehow 
(a)
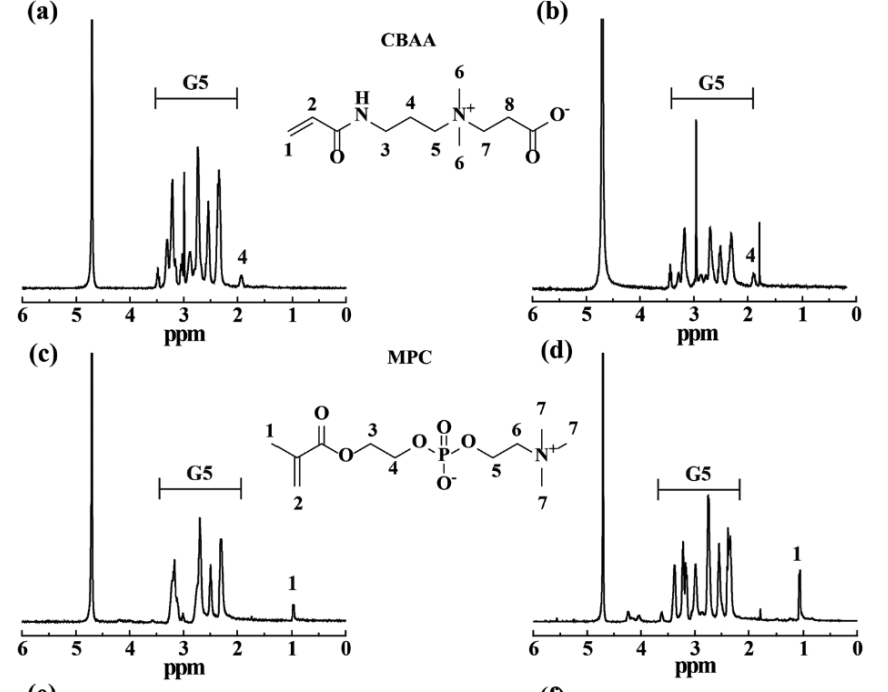

(e)
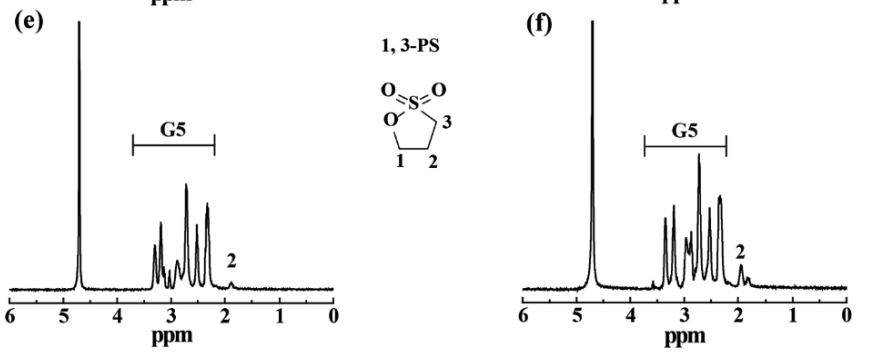

Fig. 2. ${ }^{1} \mathrm{H}$ NMR spectra of $\mathrm{G} 5 \cdot \mathrm{NH}_{2}-\mathrm{CBAA}_{20}$ (a), G5.NH ${ }_{2}^{-}$ $\mathrm{CBAA}_{80}$ (b), G5.NH $-\mathrm{MPC}_{20}$ (c), G5.NH $\mathrm{NH}_{2}-\mathrm{MPC}_{80}$ (d), G5. $\mathrm{NH}_{2}-\mathrm{PS}_{20}(\mathrm{e})$, and $\mathrm{G} 5 . \mathrm{NH}_{2}-\mathrm{PS}_{80}$ (f), respectively.

an overlap between the acetyl protons and the CBAA and 1,3-PS $-\mathrm{CH}_{2}$ - protons, the sharp peaks of acetyl protons are prominent.

\subsection{D COSY and DOSY}

The acetylated dendrimers were further characterized by 2D COSY (fig. 4) and DOSY (fig. 5). As for G5.NHAc$\mathrm{CBAA}_{20}$ and G5.NHAc-CBAA 80 dendrimers, the cross peaks in the frames may be between $\mathrm{CH}_{2}$, formed on CBAA after the addition, and $\mathrm{CH}_{2}-\mathrm{NH}$ - of the end groups of G5 dendrimer. In other words, scalar coupling between the two $\mathbf{H}$ protons of $\mathrm{OC}-\mathrm{CH}_{2}-\mathrm{CH}_{2}-\mathrm{NH}-\mathrm{CH}_{2}$ - units occurs. As for G5.NHAc-MPC 20 and G5.NHAc-MPC 80 dendrimers, the newly formed $\mathbf{H}_{2} \mathrm{C}-\mathrm{NH}-\mathrm{CH}_{2}$ - unit cannot be seen because it is under the dendrimer peaks. Probably it is in the red square, and the cross peaks are also under the dendrimer (b) $\mathrm{CH}_{2}-(\mathrm{d}) \mathrm{CH}_{2}$ cross peaks in green squares.

There are no cross peaks between the dendrimer peaks and the 1,3-PS peaks. After incorporating the 1,3-PS into the dendrimers, the formed new unit is (b) $\mathbf{H}_{2} \mathrm{C}-\mathrm{N}^{+} \ldots{ }^{-} \mathrm{O}-\mathrm{SO}_{2}-\mathrm{CH}_{2}(\gamma)$. In this unit the distance between the (b) proton of dendrimer and the $(\gamma)$ proton of 1,3 -PS is 6 bonds. It is too much for scalar coupling, therefore no scalar coupling can be expected.

The 2D DOSY spectra were also collected to confirm whether the zwitterions and G5 dendrimers were tightly
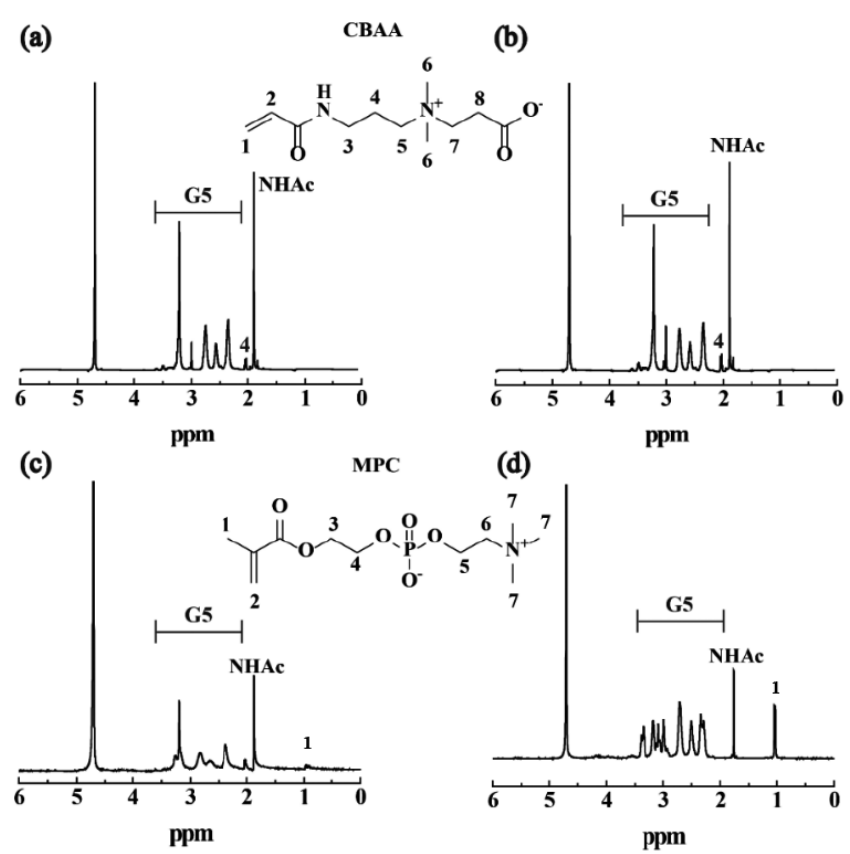

(d)
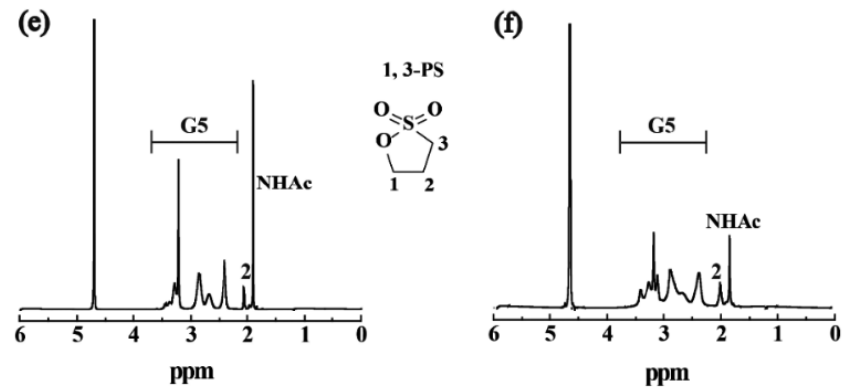

Fig. 3. ${ }^{1} \mathrm{H}$ NMR spectra of G5.NHAc-CBAA 20 (a), G5.NHAc$\mathrm{CBAA}_{80}$ (b), G5.NHAc-MPC 20 (c), G5.NHAc-MPC 80 (d), G5.NHAc-PS 20 (e), and G5.NHAc-PS 80 (f), respectively.

connected into a whole by chemical bonds. If a part of the zwitterion is not chemically bonded to the surface of the dendrimer but is physically combined with the dendrimer, the migration speed of the two parts will be different when it moves in the aqueous solution. Thus, there will be two lines in 2D DOSY spectrum except the water. The same situation is applied for diffusion coefficient. If a part of zwitterions is physically combined with the dendrimers, there would be two diffusion coefficients: one is zwitterionmodified dendrimers and the other is free zwitterions since the physical combination is not strong enough through the migration. In each of these six 2D DOSY spectra, there are only two lines, the below one represents water because water molecule moves faster than dendrimers. It means that all zwitterions are modified onto the surface of G5 dendrimers by covalent chemical bonds.

\subsection{NMR diffusiometry}

NMR diffusimetry was performed to analyze the diffusion rate of G5.NHAc-CBAA 20 , G5.NHAc-CBAA 80 , 

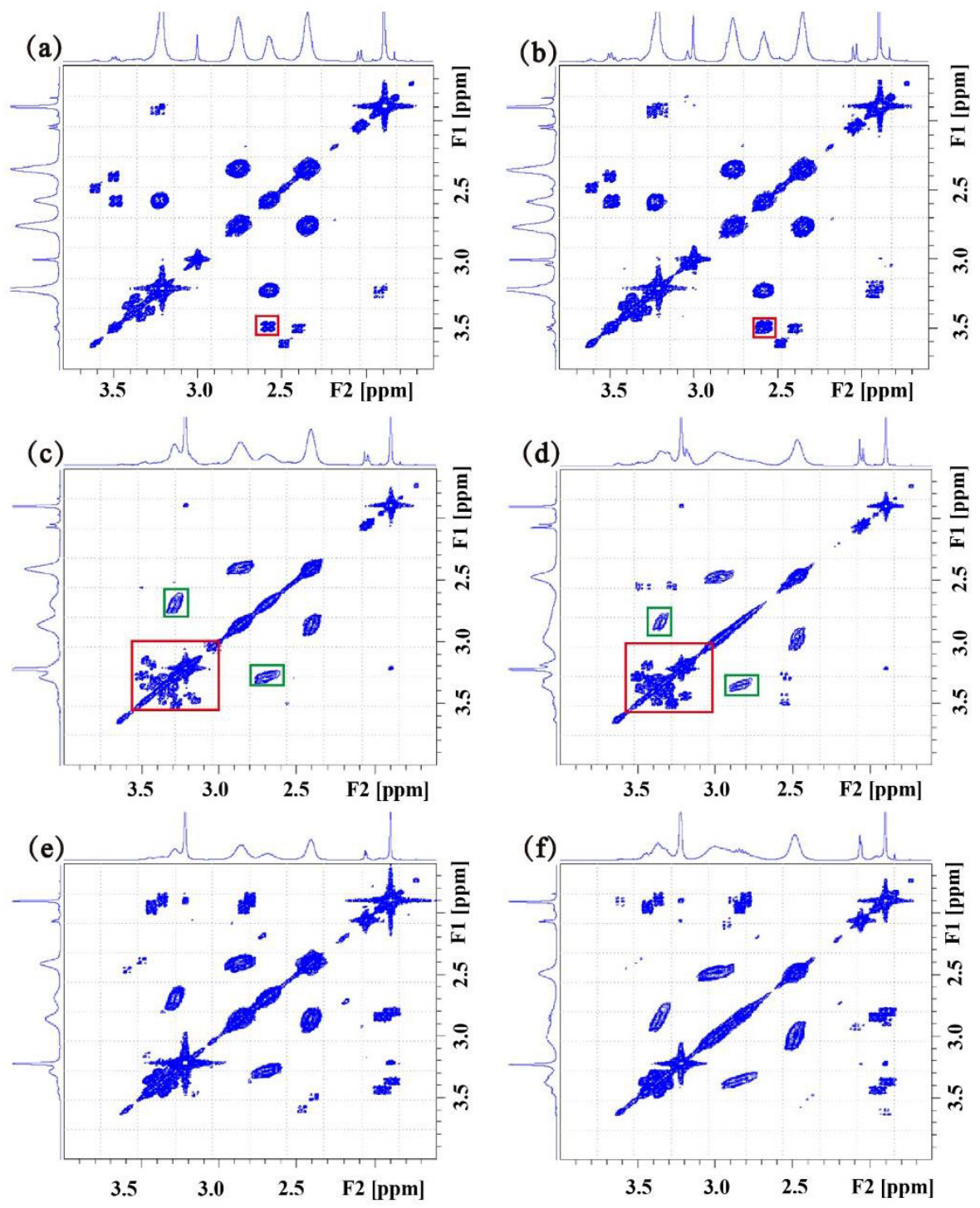

Fig. 4. $2 \mathrm{D}$ COSY spectra of G5.NHAc-CBAA 20 (a), G5.NHAc-CBAA 80 (b), G5.NHAc-MPC 20 (c), G5.NHAc-MPC 80 (d), G5.NHAc-PS 20 (e), and G5.NHAc-PS 80 (f), respectively.

G5.NHAc-MPC 20 , G5.NHAc-MPC 80 , G5.NHAc-PS 20 , and G5.NHAc-PS 80 . The diffusion time of zwitterionmodified dendrimers could be obtained from fig. 5. Furthermore, if we supposed that zwitterions were modified on the surface of spherical dendrimers equably, the hydrodynamic sizes of the zwitterionic dendrimers could be measured based on the Stokes-Einstein equation [33]:

$$
R_{H}=\frac{k_{B} T}{6 \pi \eta D},
$$

where $R_{H}$ is the hydrodynamic radius, $\kappa_{B}$ is the Boltzmann constant, $T$ is the temperature $(\mathrm{K}), \eta$ is the viscosity of deuterated water $\left(\sim 9.7 \mathrm{cP}\right.$ for $\mathrm{D}_{2} \mathrm{O}$ at $\left.30^{\circ} \mathrm{C}\right)$, and $D$ is the measured diffusion coefficient, respectively. The calculated data were shown in table 3. Apparently, zwitterion modification was able to increase the hydrodynamic size of G5 dendrimers regardless of the zwitterion types when compared to fully acetylated G5 dendrimers. For the zwitterions of CBAA and MPC, the higher modification degree on the surface of G5 dendrimers led to the larger hydrodynamic size of the G5 dendrimers. For instance, G5.NHAc-CBAA 80 dendrimer displayed a hydrodynamic radius $(3.40 \mathrm{~nm})$ slightly higher than that of G5.NHAc$\mathrm{CBAA}_{20}$ dendrimer $(3.36 \mathrm{~nm})$. As for 1,3-PS-modified G5 dendrimers, G5.NHAc-PS 80 had a slightly smaller hydrodynamic radius $(3.15 \mathrm{~nm})$ than the $\mathrm{G} 5 . \mathrm{NHAc}-\mathrm{PS}_{20}$ dendrimer $(3.27 \mathrm{~nm})$, which might be due to the short carbon chain structure different from CBAA and MPC, thus leading to the relatively compacted structure after a higher degree of modification.

\section{Conclusion}

In summary, we used NMR techniques to systematically characterize three types of zwitterion-modified G5 dendrimers with different modification degrees. We show that 

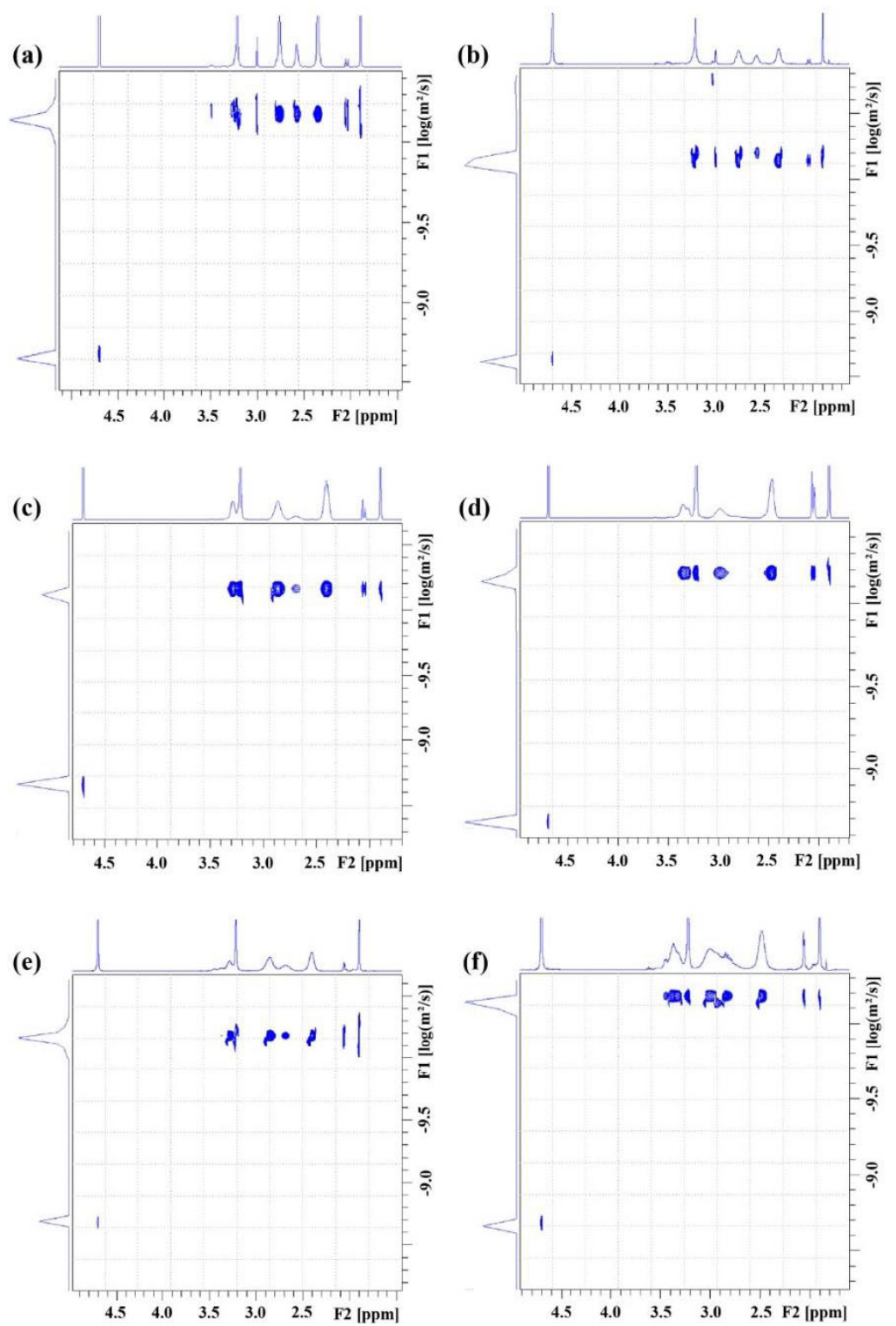

Fig. 5. $2 \mathrm{D}$ DOSY spectra of G5.NHAc-CBAA 20 (a), G5.NHAc-CBAA 80 (b), G5.NHAc-MPC 20 (c), G5.NHAc-MPC 80 (d), G5.NHAc-PS 20 (e), and G5.NHAc-PS 80 (f), respectively.

Table 3. Hydrodynamic sizes of three kinds of zwitterion-modified G5 dendrimers.

\begin{tabular}{cccc}
\hline Sample & $\begin{array}{c}D \text { (Sample }) \\
\left(\times 10^{-11} \mathrm{~m}^{2} \mathrm{~s}^{-1}\right)\end{array}$ & $\begin{array}{c}D \text { (Water }) \\
\left(\times 10^{-11} \mathrm{~m}^{2} \mathrm{~s}^{-1}\right)\end{array}$ & $R_{H}(\mathrm{~nm})$ \\
\hline $\mathrm{D}_{2} \mathrm{O}$ & & 2.02 & 3.12 \\
G5.NHAc & 6.40 & 1.94 & 3.36 \\
G5.NHAc-CBAA $_{20}$ & 5.93 & 1.93 & 3.40 \\
G5.NHAc-CBAA & 1.98 & 3.16 \\
G5.NHAc-MPC $_{20}$ & 5.86 & 1.95 & 3.33 \\
G5.NHAc-MPC $_{80}$ & 6.32 & 1.93 & 3.27 \\
G5.NHAc-PS & 5.99 & 1.90 & 3.15 \\
G5.NHAc-PS & 6.10 & 1.91 & \\
\hline
\end{tabular}


all of these three kinds of zwitterions can be linked to the surface of G5 dendrimers through covalent chemical bonds. In addition, the hydrodynamic size of zwitterionmodified G5 dendrimers can be determined by NMR diffusion. The thorough NMR structural characterization along with the hydrodynamic size measurement provide insight for their solution behaviour, which is beneficial to understand their antifouling performance for biomedical applications.

Open Access funding provided by University of Debrecen (DE). This study was financially supported by the Science and Technology Commission of Shanghai Municipality (17540712000 and 19XD1400100) and the National Natural Science Foundation of China (81761148028 and 21773026).

\section{Conflicts of interest}

There are no conflicts of interest to declare.

\section{Author contribution statement}

Xiangyang Shi designed the whole research, Jinyuan Liu and Zhijuan Xiong performed experiments, Istvan Banyai, Jinyuan Liu and Mingwu Shen analyzed the data, and all authors contributed to the writing and revisions.

Publisher's Note The EPJ Publishers remain neutral with regard to jurisdictional claims in published maps and institutional affiliations.

Open Access This is an open access article distributed under the terms of the Creative Commons Attribution License (http://creativecommons.org/licenses/by/4.0), which permits unrestricted use, distribution, and reproduction in any medium, provided the original work is properly cited.

\section{References}

1. J.A. Callow, M.E. Callow, Nat. Commun. 2, 244 (2011).

2. S.Y. Jiang, Z.Q. Cao, Adv. Mater. 22, 920 (2010).

3. L. Mi, S.Y. Jiang, Angew. Chem. Int. Ed. 53, 1746 (2014).

4. S.F. Chen, S.Y. Jiang, Adv. Mater. 20, 335 (2008).

5. Q. Shao, S.Y. Jiang, Adv. Mater. 27, 15 (2015).

6. W. Yang, H. Xue, W. Li, J.L. Zhang, S.Y. Jiang, Langmuir 25, 11911 (2009).

7. E. Chocholova, T. Bertok, L. Lorencova, A. Holazova, P. Farkas, A. Vikartovska, V. Bella, D. Velicova, P. Kasak, A.A. Eckstein, J. Mosnacek, D. Hasko, J. Tkac, Sens. Actuator B-Chem. 272, 626 (2018).
8. J. Wu, Y. Lin, H. Li, Q. Jin, J. Ji, J. Colloid Interface Sci. 485, 251 (2017).

9. H. Wei, N. Insin, J. Lee, H.S. Han, J.M. Cordero, W.H. Liu, M.G. Bawendi, Nano Lett. 12, 22 (2012).

10. S.F. Chen, J. Zheng, L.Y. Li, S.Y. Jiang, J. Am. Chem. Soc. 127, 14473 (2005).

11. Z.J. Xiong, M.W. Shen, X.Y. Shi, Bioconjug. Chem. 30, 2519 (2019)

12. A.L. Lewis, Colloids Surf. B 18, 261 (2000).

13. Y.K. Gong, F.M. Winnik, Nanoscale 4, 360 (2012).

14. Z.Q. Cao, S.Y. Jiang, Nano Today 7, 404 (2012).

15. H.W. Chien, X.W. Xu, J.R. Ella-Menye, W.B. Tsai, S.Y. Jiang, Langmuir 28, 17778 (2012).

16. J. Wu, W.F. Lin, Z. Wang, S.F. Chen, Y. Chang, Langmuir 28, 7436 (2012).

17. X.Y. Zhai, W. Wang, C.D. Wang, Q. Wang, W.G. Liu, J. Mater. Chem. 22, 23576 (2012).

18. P. Wang, J. Yang, B.Q. Zhou, Y. Hu, L.X. Xing, F.L. Xu, M.W. Shen, G.X. Zhang, X.Y. Shi, ACS Appl. Mater. Interfaces 9, 47 (2016).

19. D. Ma, J.W. Chen, Y. Luo, H. Wang, X.Y. Shi, J. Mater. Chem. B 5, 7267 (2017).

20. Z.J. Xiong, C.S. Alves, J.H. Wang, A.J. Li, J.Y. Liu, M.W. Shen, J. Rodrigues, H. Tomas, X.Y. Shi, Acta Biomater. 99, 320 (2019).

21. I. Bányai, New J. Chem. 42, 7569 (2018).

22. I. Bányai, I. Lakatos, G. Meier, Z. Nagy, S. Vass, Bull. Chem. Soc. Jpn. 90, 854 (2017).

23. M. Kéri, C. Peng, X.Y. Shi, I. Bányai, J. Phys. Chem. B 119, 3312 (2015).

24. M.E. Zhang, R. Guo, M. Kéri, I. Bányai, Y. Zheng, M. Cao, X.Y. Cao, X.Y. Shi, J. Phys. Chem. B 118, 1696 (2014).

25. S.K. Choi, T.P. Thomas, P. Leroueil, A. Kotlyar, A.F. Van Der Spek, J.R. Baker jr., J. Phys. Chem. B 116, 10387 (2012).

26. B.Q. Zhou, M.W. Shen, I. Bányai, X.Y. Shi, Analyst 141, $5390(2016)$.

27. D. Li, Y. Fan, M.W. Shen, I. Bányai, X.Y. Shi, J. Mater. Chem. B 7, 277 (2019).

28. Z.J. Xiong, Y. Wang, J.Y. Zhu, X. Li, Y. He, J. Qu, M.W. Shen, J.D. Xia, X.Y. Shi, Nanoscale 9, 12295 (2017).

29. J.Y. Liu, Z.J. Xiong, J.L. Zhang, C. Peng, B. KlajnertMaculewicz, M.W. Shen, X.Y. Shi, ACS Appl. Mater. Interfaces 11, 15212 (2019).

30. B. Yu, A.B. Lowe, K. Ishihara, Biomacromolecules 10, 950 (2009).

31. Y.W. Pei, A.B. Lowe, Polym. Chem. 5, 2342 (2014).

32. C. Peng, L.F. Zheng, Q. Chen, M.W. Shen, R. Guo, H. Wang, X.Y. Cao, G.X. Zhang, X.Y. Shi, Biomaterials 33, 1107 (2012).

33. A. Macchioni, G. Ciancaleoni, C. Zuccaccia, D. Zuccaccia, Chem. Soc. Rev. 37, 479 (2008). 